\title{
On exceptional times for pointwise convergence of integral kernels in Feynman-Trotter path integrals
}

\author{
Hans G. Feichtinger, Fabio Nicola, and S. Ivan Trapasso
}

\begin{abstract}
In the first part of the paper we provide a survey of recent results concerning the problem of pointwise convergence of integral kernels in Feynman path integral, obtained by means of time-frequency analysis techniques. We then focus on exceptional times, where the previous results do not hold, and we show that weaker forms of convergence still occur. In conclusion we offer some clues about possible physical interpretation of exceptional times.
\end{abstract}

\section{Introduction}

Integration over infinite-dimensional spaces of paths plays a relevant role in modern quantum physics. This machinery first appeared in a 1948 paper [21] by Richard Feynman, shortly followed by [22] where path integrals paved the way to the celebrated Feynman diagrams, hence to a completely new way to investigate field theories.

Let us briefly recall the most important features of the functional integral formulation of (non-relativistic) quantum mechanics. The interested reader may consult the textbook [23] for a comprehensive introduction to the subject from a physical perspective. Recall that the state of a particle in $\mathbb{R}^{d}$ at time $t \in \mathbb{R}$ is represented by the wave function $\psi(t, x),(t, x) \in \mathbb{R} \times \mathbb{R}^{d}$, such that $\psi(t, \cdot) \in L^{2}\left(\mathbb{R}^{d}\right)$. The time

Hans G. Feichtinger

Faculty of Mathematics, University of Vienna, Oskar-Morgenstern-Platz 1, A-1090 Wien.

e-mail: hans.feichtinger@univie.ac.at

Fabio Nicola

Dipartimento di Scienze Matematiche "G. L. Lagrange", Politecnico di Torino,

Corso Duca degli Abruzzi 24, 10129, Torino. e-mail: fabio.nicola@polito.it

S. Ivan Trapasso

Dipartimento di Scienze Matematiche "G. L. Lagrange", Politecnico di Torino,

Corso Duca degli Abruzzi 24, 10129, Torino. e-mail: salvatore.trapasso@polito.it 
evolution of the initial state $\varphi(x)$ at $t=0$ is regulated by the corresponding Cauchy problem for the Schrödinger equation:

$$
\left\{\begin{array}{l}
i \hbar \partial_{t} \psi=\left(H_{0}+V(x)\right) \psi \\
\psi(0, x)=\varphi(x),
\end{array}\right.
$$

where $0<\hbar \leq 1$ is a parameter (representing the Planck constant), $H_{0}=-\hbar^{2} \Delta / 2$ is the free particle Hamiltonian and $V$ is a real-valued potential; we set $m=1$ for the mass of the particle. The map $U(t, s): \psi(s, \cdot) \mapsto \psi(t, \cdot), t, s \in \mathbb{R}$, is a unitary operator on $L^{2}\left(\mathbb{R}^{d}\right)$ and is called propagator or evolution operator; we set $U(t)$ for $U(t, 0)$. Since $U(t)$ is a linear operator we may formally represent it as an integral operator with distribution kernel $u_{t}$, namely

$$
\psi(t, x)=\int_{\mathbb{R}^{d}} u_{t}(x, y) \varphi(y) d y .
$$

The kernel $u_{t}$ (actually known as propagator in physics) is interpreted as the transition amplitude from the position $y$ at time 0 to the position $x$ at time $t$. In his papers Feynman essentially provided a recipe for how to compute this kernel, involving all the possible interfering alternative paths from $y$ to $x$ that could be followed by the particle. In particular, each path would contribute to the total probability amplitude with a phase factor proportional to the action functional corresponding to the path:

$$
S[\gamma]=S(t, 0, x, y)=\int_{s}^{t} L(\gamma(\tau), \dot{\gamma}(\tau)) d \tau,
$$

where $L$ is the Lagrangian of the corresponding classical system. In a nutshell, a formal representation of the kernel is

$$
u_{t}(x, y)=\int e^{\frac{i}{\hbar} S[\gamma]} \mathcal{D} \gamma,
$$

underpinning some integration procedure over the infinite-dimensional space of paths satisfying the conditions above. Notice that (a still formal) application of the stationary phase principle shows that the semiclassical limit $\hbar \rightarrow 0$ selects the classical trajectory, in according with the principle of stationary action of classical mechanics.

\subsection{The mathematics of path integrals}

In spite of the popularity and the successful predictions of path integrals, it is not clear what the meaning of (2) could be from a mathematical point of view. This is in fact an open subfield of functional analysis and there have been several attempts to provide a rigorous and satisfactory theory of path integrals with the support of 
techniques ranging from infinite-dimensional analysis to operator theory, but also from stochastics to geometry. We cannot hope to frame here more than seventy years of literature; we suggest the monographs [1, 27, 37, 39] as points of departure as well as the article [2] for a broad overview. We remark that only in recent times techniques from time-frequency analysis have been fruitfully used in the study of mathematical path integrals, see for instance [41, 43, 44]; see also [50] for an expository paper on the topic.

Among the several frameworks mentioned above we focus here on the so-called sequential approach, introduced by Nelson in [40]. The reasons behind this choice are manifold; first, it is probably the mathematical scheme which best meets Feynman's original insight and some of its features are nowadays part of the custom in physics literature, cf. [32, 38]. Moreover, the perturbative nature of this approach is very well suited to certain function spaces and operators related to time-frequency analysis, as will be elucidated later.

Nelson's approach relies on two issues. Recall that the evolution operator for the Schrödinger equation with $V=0$, namely $U_{0}(t)=e^{-\frac{i}{\hbar} t H_{0}}, H_{0}=-\hbar^{2} \Delta / 2$, is a Fourier multiplier; an explicit representation can be derived after standard computation (cf. [46, Sec. IX.7]):

$$
e^{-\frac{i}{\hbar} t H_{0}} \varphi(x)=\frac{1}{(2 \pi i t \hbar)^{d / 2}} \int_{\mathbb{R}^{d}} \exp \left(\frac{i}{\hbar} \frac{|x-y|^{2}}{2 t}\right) \varphi(y) d y, \quad \varphi \in \mathcal{S}\left(\mathbb{R}^{d}\right) .
$$

The second ingredient is a well-known tool from the theory of operator semigroups. Under suitable conditions on the domain of $H_{0}$ and on the potential 2 , the Trotter product formula holds for the semigroup generated by $H=H_{0}+V$, namely it can be expressed in terms of a strong operator limit in $L^{2}\left(\mathbb{R}^{d}\right)$ :

$$
e^{-\frac{i}{\hbar} t\left(H_{0}+V\right)}=\lim _{n \rightarrow \infty}\left(e^{-\frac{i}{\hbar} \frac{t}{n} H_{0}} e^{-\frac{i}{\hbar} \frac{t}{n} V}\right)^{n} .
$$

The joint application of these two results gives that the complete propagator $e^{-\frac{i}{\hbar} t H}$ can be expressed as limit of integral operators (cf. [46, Thm. X.66]):

$$
e^{-\frac{i}{\hbar} t\left(H_{0}+V\right)} \varphi(x)=\lim _{n \rightarrow \infty}\left(2 \pi \hbar i \frac{t}{n}\right)^{-\frac{n d}{2}} \int_{\mathbb{R}^{n d}} e^{\frac{i}{\hbar} S_{n}\left(t ; x_{0}, \ldots, x_{n-1}, x\right)} \varphi\left(x_{0}\right) d x_{0} \ldots d x_{n-1},
$$

where we set

$$
S_{n}\left(t ; x_{0}, \ldots, x_{n-1}, x\right)=\sum_{k=1}^{n} \frac{t}{n}\left[\frac{1}{2}\left(\frac{\left|x_{k}-x_{k-1}\right|}{t / n}\right)^{2}-V\left(x_{k}\right)\right], \quad x_{0}=y, x_{n}=x .
$$

The role of the phase $S_{n}\left(t ; x_{0}, \ldots, x_{n}\right)$ may be clarified by the following argument: given the points $x_{0}, \ldots, x_{n-1}, x \in \mathbb{R}^{d}$, let $\bar{\gamma}$ be the polygonal path through the vertices

${ }^{1}$ For instance one may consider a potential $V$ such that $H_{0}+V$ is essentially self-adjoint on $D\left(H_{0}\right) \cap D(V)$, cf. [45 Sec. VIII.8]. 


$$
\begin{aligned}
& x_{k}=\bar{\gamma}(k t / n), k=0, \ldots, n, x_{n}=x, \text { parametrized as } \\
& \bar{\gamma}(\tau)=x_{k}+\frac{x_{k+1}-x_{k}}{t / n}\left(\tau-k \frac{t}{n}\right), \quad \tau \in\left[k \frac{t}{n},(k+1) \frac{t}{n}\right], \quad k=0, \ldots, n-1 .
\end{aligned}
$$

Hence $\bar{\gamma}$ prescribes a classical motion with constant velocity along each segment. The action functional for such path is given by

$$
S[\bar{\gamma}]=\sum_{k=1}^{n} \frac{1}{2} \frac{t}{n}\left(\frac{\left|x_{k}-x_{k-1}\right|}{t / n}\right)^{2}-\int_{0}^{t} V(\bar{\gamma}(\tau)) d \tau .
$$

According to Feynman's heuristics, the relation in (4) should be interpreted as the definition of an integral over all polygonal paths while $S_{n}\left(x_{0}, \ldots, x_{n}, t\right)$ is a Riemannlike, finite-dimensional approximation of the action functional evaluated on them. The regime $n \rightarrow \infty$ is then intuitively clear: the set of polygonal paths becomes the set of all paths and we recover (2).

\subsection{Convergence at the level of integral kernels}

The sequential approach discussed above seems to suggest that path integral can be made mathematically rigorous at the level of operators rather than integral kernels. This remark is reinforced by the achievements of different mathematical theories of path integrals relying on the standard operator-theoretic approach to quantum mechanics. Consider for instance the so-called time slicing approximation approach introduced by Fujiwara in celebrated papers like [25, 26] - see also the monograph [27] for a systematic exposition; broadly speaking, the philosophy underlying these works is to design sequences of finite-dimensional approximation operators on $L^{2}\left(\mathbb{R}^{d}\right)$ (in particular, oscillatory integral operators) and then prove convergence to the exact propagator $U(t)$ in some operator topology on $L^{2}$.

Actually, there are good reasons for not being completely satisfied with this state of affairs. The lesson of Feynman's original formulation strongly motivates a focus shift from operators to their kernels, in particular to the problem of pointwise convergence of the integral kernels in (4) to the kernel $u_{t}$ of the propagator. This may appear as an unaffordable problem in general since non-regular or even purely distribution kernels may show up, thus the problem of convergence can be hard or even pointless. A strong clue pointing in this direction comes from the already mentioned papers by Fujiwara, where convergence in a finer topology at the level of integral kernels is proved for sufficiently small time intervals and smooth potentials with at most quadratic growth.

We describe below the recent results obtained by two of the authors in [43], where techniques of time-frequency analysis are fruitfully used to prove pointwise convergence of integral kernels in the framework provided by the sequential approach. In contrast with the aforementioned results by Fujiwara we consider bounded potentials 
(the minimal regularity assumption is continuity) and we obtain the desired convergence for the kernels in suitable topologies which imply pointwise convergence. Our results are global in time, namely they hold for any fixed $t \in \mathbb{R} \backslash \widetilde{E}$, where $\widetilde{E}$ is a set of exceptional times. We describe below the most important features of this set from both the mathematical and physical points of view and provide explicit examples. For the moment we confine ourselves to remark that exceptional times are to be expected: recall that the involved kernels are in general tempered distributions in $\mathcal{S}^{\prime}\left(\mathbb{R}^{d}\right)$ in view of the Schwartz kernel theorem and the problem of pointwise convergence is well-posed only when the kernels are actually functions. One may still wonder whether there is convergence at exceptional times in some weaker distribution sense. We are able to prove global-in-time convergence in this fashion, again supported by the framework of time-frequency analysis techniques and function spaces. In order to precisely state and prove the claimed results we devote the next section to collect some preparatory material.

\section{Preliminaries}

\subsection{Notation}

We set $x^{2}=x \cdot x$, for $x \in \mathbb{R}^{d}$, where $x \cdot y$ is the scalar product on $\mathbb{R}^{d}$. The Schwartz class is denoted by $\mathcal{S}\left(\mathbb{R}^{d}\right)$, the space of tempered distributions by $\mathcal{S}^{\prime}\left(\mathbb{R}^{d}\right)$. The brackets $\langle f, g\rangle$ denote the extension to $\mathcal{S}^{\prime}\left(\mathbb{R}^{d}\right) \times \mathcal{S}\left(\mathbb{R}^{d}\right)$ of the inner product $\langle f, g\rangle=\int_{\mathbb{R}^{d}} f(x) \overline{g(x)} d x$ on $L^{2}\left(\mathbb{R}^{d}\right)$, but also other related dualities described below.

The conjugate exponent $p^{\prime}$ of $p \in[1, \infty]$ is defined by $1 / p+1 / p^{\prime}=1$. The symbol $\lesssim$ means that the underlying inequality holds up to a positive constant factor $C>0$. For any $x \in \mathbb{R}^{d}$ and $s \in \mathbb{R}$ we set $\langle x\rangle^{s}:=\left(1+|x|^{2}\right)^{s / 2}$. We choose the following normalization for the Fourier transform:

$$
\mathcal{F} f(\xi)=\int_{\mathbb{R}^{d}} e^{-2 \pi i x \cdot \xi} f(x) d x, \quad \xi \in \mathbb{R}^{d} .
$$

We define the translation and modulation operators: for any $x, \xi \in \mathbb{R}^{d}$ and $f \in \mathcal{S}\left(\mathbb{R}^{d}\right)$,

$$
\left(T_{x} f\right)(y):=f(y-x), \quad\left(M_{\xi} f\right)(y):=e^{2 \pi i \xi \cdot y} f(y) .
$$

These operators can be extended by duality on tempered distributions. The composition $\pi(x, \xi)=M_{\xi} T_{x}$ constitutes a so-called time-frequency shift.

Given a normed linear space of distributions $X \subset \mathcal{S}^{\prime}\left(\mathbb{R}^{d}\right)$, we set

$$
\begin{gathered}
X_{\text {comp }}:=\left\{u \in X: \operatorname{supp}(u) \text { is a compact subset of } \mathbb{R}^{d}\right\}, \\
X_{\mathrm{loc}}:=\left\{u \in \mathcal{S}^{\prime}\left(\mathbb{R}^{d}\right): \phi u \in X \forall \phi \in C_{c}^{\infty}\left(\mathbb{R}^{d}\right)\right\} .
\end{gathered}
$$


In the rest of the paper we set $\hbar=1$ for convenience, since we are not concerned with semiclassical aspects.

\subsection{Modulation spaces}

The short-time Fourier transform (STFT) of a tempered distribution $f \in \mathcal{S}^{\prime}\left(\mathbb{R}^{d}\right)$ with respect to the window function $g \in \mathcal{S}\left(\mathbb{R}^{d}\right) \backslash\{0\}$ is defined by

$$
V_{g} f(x, \xi):=\langle f, \pi(x, \xi) g\rangle=\mathcal{F}\left(f \cdot T_{x} g\right)(\xi)=\int_{\mathbb{R}^{d}} e^{-2 \pi i y \cdot \xi} f(y) \overline{g(y-x)} d y .
$$

The monograph [28] contains a comprehensive treatment of the mathematical properties of this time-frequency representation, especially those mentioned below. We stress that the STFT is deeply connected with other well-known phase-space transforms, in particular the Wigner distribution

$$
W(f, g)(x, \xi)=\int_{\mathbb{R}^{d}} e^{-2 \pi i y \cdot \xi} f\left(x+\frac{y}{2}\right) \overline{g\left(x-\frac{y}{2}\right)} d y .
$$

Given a non-zero window $g \in \mathcal{S}\left(\mathbb{R}^{d}\right), s \in \mathbb{R}$ and $1 \leq p, q \leq \infty$, the modulation space $M_{s}^{p, q}\left(\mathbb{R}^{d}\right)$ consists of all tempered distributions $f \in \mathcal{S}^{\prime}\left(\mathbb{R}^{d}\right)$ such that $V_{g} f \in$ $L_{s}^{p, q}\left(\mathbb{R}^{2 d}\right)$ (mixed weighted Lebesgue space), that is:

$$
\|f\|_{M_{s}^{p, q}}=\left\|V_{g} f\right\|_{L_{s}^{p, q}}=\left(\int_{\mathbb{R}^{d}}\left(\int_{\mathbb{R}^{d}}\left|V_{g} f(x, \xi)\right|^{p} d x\right)^{q / p}\langle\xi\rangle^{q s} d \xi\right)^{1 / q}<\infty
$$

with trivial modification if $p$ or $q$ is $\infty$. If $p=q$, we write $M^{p}$ instead of $M^{p, p}$, while for the unweighted case $(s=0)$ we set $M_{0}^{p, q} \equiv M^{p, q}$.

It can be proved that $M_{s}^{p, q}\left(\mathbb{R}^{d}\right)$ is a Banach space whose definition does not depend on the choice of the window $g$. We mention that many common function spaces are intimately related with modulation spaces: for instance,

(i) $M^{2}\left(\mathbb{R}^{d}\right)$ coincides with the Hilbert space $L^{2}\left(\mathbb{R}^{d}\right)$;

(ii) $M_{s}^{2}\left(\mathbb{R}^{d}\right)$ coincides with the usual $L^{2}$-based Sobolev space $H^{s}\left(\mathbb{R}^{d}\right)$;

(iii) the following continuous embeddings with Lebesgue spaces hold:

$$
M_{r}^{p, q}\left(\mathbb{R}^{d}\right) \hookrightarrow L^{p}\left(\mathbb{R}^{d}\right) \hookrightarrow M_{s}^{p, q}\left(\mathbb{R}^{d}\right), \quad r>d / q^{\prime} \text { and } s<-d / q .
$$

In particular,

$$
M^{p, 1}\left(\mathbb{R}^{d}\right) \hookrightarrow L^{p}\left(\mathbb{R}^{d}\right) \hookrightarrow M^{p, \infty}\left(\mathbb{R}^{d}\right) .
$$

For these and other embeddings we address the reader to [13, 15, 16, 28].

We wish to focus on distinguished members of the family of modulation spaces. The Banach-Gelfand triple $\left(M^{1}\left(\mathbb{R}^{d}\right), L^{2}\left(\mathbb{R}^{d}\right), M^{\infty}\left(\mathbb{R}^{d}\right)\right)$ proved to be a very fruitful generalization of the standard triple $\left(\mathcal{S}\left(\mathbb{R}^{d}\right), L^{2}\left(\mathbb{R}^{d}\right), \mathcal{S}^{\prime}\left(\mathbb{R}^{d}\right)\right)$ for the purposes of 
time-frequency analysis, see [3, 20, 35] for further details. The space $M^{1}\left(\mathbb{R}^{d}\right)$ is also known as the Feichtinger algebra [13] and it does enjoy a large number of particularly nice properties. We stress that $\mathcal{S}\left(\mathbb{R}^{d}\right) \subset M^{1}\left(\mathbb{R}^{d}\right)$ and $L^{2}\left(\mathbb{R}^{d}\right)$ is the completion of $M^{1}\left(\mathbb{R}^{d}\right)$ with respect to $\|\cdot\|_{L^{2}}$ norm. Moreover $\left(M^{1}\left(\mathbb{R}^{d}\right)\right)^{\prime}=M^{\infty}\left(\mathbb{R}^{d}\right)$ under the duality

$$
\langle f, \phi\rangle=\int_{\mathbb{R}^{2 d}} V_{g} f(z) \overline{V_{g} \phi}(z) d z, \quad f \in M^{1}\left(\mathbb{R}^{d}\right), \phi \in M^{\infty}\left(\mathbb{R}^{d}\right),
$$

for any $g \in \mathcal{S}\left(\mathbb{R}^{d}\right) \backslash\{0\}$, without loss of generality with $\|g\|_{2}=1$. Finally, $M^{1}\left(\mathbb{R}^{d}\right)$ is isometrically invariant under Fourier transform and arbitrary time-frequency shifts, and the embedding $M^{1}\left(\mathbb{R}^{d}\right) \hookrightarrow M^{p, q}\left(\mathbb{R}^{d}\right)$ hold for all $1 \leq p, q \leq \infty$. An additional benefit of this extended framework is that one may derive a streamlined and selfconsistent presentation of the mathematical foundations of signal analysis with a limited amount of technicalities, cf. [19].

The role of $\left(M^{1}, L^{2}, M^{\infty}\right)$ as a Gelfand triple is further reinforced by the $\mathrm{Fe}$ ichtinger kernel theorem [14, 17, 18, 7].

Theorem 1 (i) Every distribution $k \in M^{\infty}\left(\mathbb{R}^{2 d}\right)$ defines a bounded linear operator $T: M^{1}\left(\mathbb{R}^{d}\right) \rightarrow M^{\infty}\left(\mathbb{R}^{d}\right)$ according to

$$
\langle T f, g\rangle=\langle k, g \otimes \bar{f}\rangle, \quad \forall f, g \in M^{1}\left(\mathbb{R}^{d}\right),
$$

with $\|T\|_{M^{1} \rightarrow M^{\infty}} \lesssim\|k\|_{M^{\infty}}$.

(ii) Any linear bounded operator $T: M^{1}\left(\mathbb{R}^{d}\right) \rightarrow M^{\infty}\left(\mathbb{R}^{d}\right)$ arises in this way for a unique kernel $k \in M^{\infty}\left(\mathbb{R}^{2 d}\right)$; moreover $\|k\|_{M^{\infty}} \lesssim\|T\|_{M^{1} \rightarrow M^{\infty}}$.

Another interesting modulation space is $M^{\infty, 1}\left(\mathbb{R}^{d}\right)$, also known as the Sjöstrand class since it was highlighted in [49] as an exotic symbol class still yielding bounded pseudodifferential operators on $L^{2}\left(\mathbb{R}^{d}\right)$ (see the next section for further details, also [29, 31]). In order to specify the regularity of functions in this space recall the definition of the Fourier-Lebesgue space: for $s \in \mathbb{R}$ we set

$$
f \in \mathcal{F} L_{s}^{1}\left(\mathbb{R}^{d}\right) \quad \Leftrightarrow \quad\|f\|_{\mathcal{F} L_{s}^{1}}=\int_{\mathbb{R}^{d}}|\mathcal{F} f(\xi)|\langle\xi\rangle^{s} d \xi<\infty .
$$

\section{Proposition 1 ([28] and [44, Prop. 3.4])}

1. $M^{\infty, 1}\left(\mathbb{R}^{d}\right) \subset\left(\mathcal{F} L^{1}\right)_{\mathrm{loc}}\left(\mathbb{R}^{d}\right) \cap L^{\infty}\left(\mathbb{R}^{d}\right) \subset C^{0}\left(\mathbb{R}^{d}\right) \cap L^{\infty}\left(\mathbb{R}^{d}\right)$.

2. $\left(M^{\infty, 1}\right)_{\mathrm{loc}}\left(\mathbb{R}^{d}\right)=\left(\mathcal{F} L^{1}\right)_{\mathrm{loc}}\left(\mathbb{R}^{d}\right)=(\mathcal{F} \mathcal{M})_{\mathrm{loc}}\left(\mathbb{R}^{d}\right)$, where $\mathcal{F} \mathcal{M}\left(\mathbb{R}^{d}\right)$ is the space of Fourier transforms of (finite) complex measures on $\mathbb{R}^{d}$.

3. $\mathcal{F} \mathcal{M}\left(\mathbb{R}^{d}\right) \subset M^{\infty, 1}\left(\mathbb{R}^{d}\right)$.

The equality $\left(\mathcal{F} L^{1}\right)_{\mathrm{loc}}\left(\mathbb{R}^{d}\right)=(\mathcal{F} \mathcal{M})_{\text {loc }}\left(\mathbb{R}^{d}\right)$ is an immediate consequence of the fact that $L^{1}\left(\mathbb{R}^{d}\right)$ is an ideal in the convolution algebra $\mathcal{M}\left(\mathbb{R}^{d}\right)$.

Moreover, $M^{\infty, 1}\left(\mathbb{R}^{d}\right)$ is a Banach algebra under pointwise product. In fact, precise conditions are known on $p, q$ and $s$ in order for $M_{s}^{p, q}$ to be a Banach algebra with respect to pointwise multiplication. 
Proposition 2 ([47, Thm. 3.5 and Cor. 2.10]) Let $1 \leq p, q \leq \infty$ and $s \in \mathbb{R}$. The following facts are equivalent.

(i) $M_{s}^{p, q}\left(\mathbb{R}^{d}\right)$ is a Banach algebra for pointwise multiplication 1

(ii) $M_{s}^{p, q}\left(\mathbb{R}^{d}\right) \hookrightarrow L^{\infty}\left(\mathbb{R}^{d}\right)$.

(iii) Either $s=0$ and $q=1$ or $s>d / q^{\prime}$.

We deduce that also the modulation spaces $M_{s}^{\infty}\left(\mathbb{R}^{d}\right)$ with $s>d$ are Banach algebras for pointwise multiplication. In particular we have $M_{s}^{\infty}\left(\mathbb{R}^{d}\right) \hookrightarrow M^{\infty, 1}\left(\mathbb{R}^{d}\right)$ for $s>d$ and the following characterization holds:

$$
C_{b}^{\infty}\left(\mathbb{R}^{d}\right):=\left\{f \in C^{\infty}\left(\mathbb{R}^{d}\right):\left|\partial^{\alpha} f\right| \leq C_{\alpha} \quad \forall \alpha \in \mathbb{N}^{d}\right\}=\bigcap_{s \geq 0} M_{s}^{\infty}\left(\mathbb{R}^{d}\right) ;
$$

see [30, Lemma 6.1] for further details.

\subsection{Weyl operators}

The success of time-frequency analysis in the theory of pseudodifferential operators mainly relies on the following equality:

$$
\left\langle\sigma^{\mathrm{w}} f, g\right\rangle=\langle\sigma, W(g, f)\rangle, \quad \forall f, g \in \mathcal{S}\left(\mathbb{R}^{d}\right),
$$

where $\sigma \in \mathcal{S}^{\prime}\left(\mathbb{R}^{2 d}\right)$ is the symbol of the Weyl operator $\sigma^{\mathrm{w}}: \mathcal{S}\left(\mathbb{R}^{d}\right) \rightarrow \mathcal{S}^{\prime}\left(\mathbb{R}^{d}\right)$, which can be formally represented as

$$
\sigma^{\mathrm{w}} f(x):=\int_{\mathbb{R}^{2 d}} e^{2 \pi i(x-y) \cdot \xi} \sigma\left(\frac{x+y}{2}, \xi\right) f(y) d y d \xi,
$$

while $W(g, f)$ is the Wigner transform defined in (7). The main benefit of a timefrequency approach to Weyl operators is that very general symbol classes may be taken into account, in particular modulation spaces - recall that classical symbol classes are usually defined by means of decay/smoothness conditions, such as the Hördmander classes $S_{\rho, \delta}^{m}\left(\mathbb{R}^{2 d}\right)$ [34]. Moreover, most of the properties of $\sigma^{\mathrm{w}}$ are intimately connected to those of the Wigner transform, the latter being very well established nowadays [11, 28].

The composition of Weyl transforms induces a bilinear form on symbols, the so-called twisted product: this means that the composition of two operators $\sigma^{\mathrm{w}} \circ \rho^{\mathrm{w}}$ is in fact a Weyl operator with special symbol denoted by $\sigma \# \rho$. Explicit formulas for $\sigma \# \rho$ are known (cf. [51]) but we are more interested in the algebra structure

${ }^{2}$ To be precise, we provide conditions under which the embedding $M_{s}^{p, q} \cdot M_{s}^{p, q} \hookrightarrow M_{S}^{p, q}$ is continuous; this means that the algebra property holds up to a constant. It is a rather standard result that that there exists an equivalent norm for which the previous estimate holds with $C=1$ (cf. [48, Thm. 10.2]). This setting will be tacitly assumed whenever concerned with Banach algebras from now on. 
induced on symbol spaces. It is indeed a peculiar feature of $M^{\infty, 1}\left(\mathbb{R}^{2 d}\right)$, as well as of $M_{s}^{\infty}\left(\mathbb{R}^{2 d}\right)$ with $s>2 d$, to enjoy a double Banach algebra structure:

- a commutative one with respect to the pointwise multiplication as a consequence of Proposition2,

- a non-commutative one with respect to the twisted product of symbols ([30, 49]); for instance, $\sigma, \rho \in M^{\infty, 1}\left(\mathbb{R}^{2 d}\right) \Longrightarrow \sigma \# \rho \in M^{\infty, 1}\left(\mathbb{R}^{2 d}\right)$.

Furthermore, it turns out that the latter algebraic structure can be related to a characterizing sparse behaviour satisfied by pseudodifferential operators with symbols in those spaces, the so-called almost diagonalization property with respect to timefrequency shifts; it can be proved that $\sigma \in M_{S}^{\infty}\left(\mathbb{R}^{2 d}\right)$ if and only if, for some (hence any) $g \in \mathcal{S}\left(\mathbb{R}^{d}\right) \backslash\{0\}$,

$$
\left|\left\langle\sigma^{w} \pi(z) g, \pi(w) g\right\rangle\right| \leq C\langle w-z\rangle^{-s}, \quad z, w \in \mathbb{R}^{2 d} .
$$

In a similar fashion, $\sigma \in M^{\infty, 1}\left(\mathbb{R}^{2 d}\right)$ if and only if there exists $H \in L^{1}\left(\mathbb{R}^{2 d}\right)$ such that

$$
\left|\left\langle\sigma^{w} \pi(z) g, \pi(w) g\right\rangle\right| \leq H(w-z), \quad z, w \in \mathbb{R}^{2 d} .
$$

The reader may consult [4, 5, 6, 10, 29, 30] for further details on this topic.

\section{Pointwise convergence of integral kernels}

The main results in [44] require us to consider a slightly generalized version of the free Hamiltonian operator $H_{0}$ in (1). Let $a$ be a quadratic homogeneous polynomial on $\mathbb{R}^{2 d}$, namely

$$
a(x, \xi)=\frac{1}{2} x \cdot A x+\xi \cdot B x+\frac{1}{2} \xi \cdot C \xi,
$$

for some symmetric matrices $A, C \in \mathbb{R}^{d \times d}$ and $B \in \mathbb{R}^{d \times d}$. The solution of (1) with $H_{0}=a^{\mathrm{w}}$ (the Weyl transform of $a$ ) and $V=0$ is given by

$$
\psi(t, x)=e^{-i t H_{0}} \varphi(x)=\mu\left(\mathcal{A}_{t}\right) \varphi(x),
$$

where $\mu\left(\mathcal{A}_{t}\right)$ is a metaplectic operator - see [11, Sec. 15.1.3] and also [8, 24] for a complete derivation of this classic result. A precise characterization of metaplectic operators would lead us too far, hence we just outline their main features. First, recall that the phase-space flow governed by the Hamilton equation 3

$$
2 \pi \dot{z}=J \nabla_{z} a(z)=\mathbb{A}, \quad \mathbb{A}=\left(\begin{array}{cc}
B & C \\
-A & -B^{\top}
\end{array}\right),
$$

defines a mapping

\footnotetext{
${ }^{3}$ The factor $2 \pi$ is a consequence of the normalization of the Fourier transform adopted in the paper.
} 


$$
\mathbb{R} \ni t \mapsto \mathcal{A}_{t}=e^{(t / 2 \pi) \mathbb{A}}=\left(\begin{array}{ll}
A_{t} & B_{t} \\
C_{t} & D_{t}
\end{array}\right) \in \operatorname{Sp}(d, \mathbb{R}) .
$$

In sloppy terms, any symplectic matrix $S \in \operatorname{Sp}(d, \mathbb{R})$ is associated with a unitary bounded operator $\mu(S)$ on $L^{2}\left(\mathbb{R}^{d}\right)$ which satisfies the intertwining property

$$
\mu(S)^{-1} \sigma^{\mathrm{w}} \mu(S)=(\sigma \circ S)^{\mathrm{w}}, \quad \sigma \in \mathcal{S}^{\prime}\left(\mathbb{R}^{2 d}\right) .
$$

In particular, the classical flow $\mathcal{A}_{t}$ is associated (up to a complex phase factor) with a family of unitary operators on $L^{2}\left(\mathbb{R}^{d}\right)$ (for details see [28], Thm. 9.4.2) An explicit formula for $\mu\left(\mathcal{A}_{t}\right)$ may be provided in some special cases: for all $t \in \mathbb{R}$ such that $\mathcal{A}_{t}$ is a free symplectic matrix, namely such that the upper-right block $B_{t}$ is invertible, the corresponding metaplectic operator may be represented as a quadratic Fourier transform [11, Sec. 7.2.2], namely

$$
\mu\left(\mathcal{A}_{t}\right) \varphi(x)=c_{t}\left|\operatorname{det} B_{t}\right|^{-1 / 2} \int_{\mathbb{R}^{d}} e^{2 \pi i \Phi_{t}}(x, \xi) \varphi(y) d y, \quad \varphi \in \mathcal{S}\left(\mathbb{R}^{d}\right),
$$

for suitable $c_{t} \in \mathbb{C},\left|c_{t}\right|=1$, where

$$
\Phi_{t}(x, y)=\frac{1}{2} x \cdot D_{t} B_{t}^{-1} x-y \cdot B_{t}^{-1} x+\frac{1}{2} y \cdot B_{t}^{-1} A_{t} y, \quad x, y \in \mathbb{R}^{d} .
$$

This representation of $\mu\left(\mathcal{A}_{t}\right)$ is a main ingredient of our results, hence we stress that it does hold for any $t \in \mathbb{R} \backslash \widetilde{E}$, where we define the set of exceptional times as

$$
\widetilde{E}=\left\{t \in \mathbb{R}: \operatorname{det} B_{t}=0\right\} .
$$

Some of the properties of this set can be immediately deduced from the fact that it is indeed the zero set of an analytic function: apart from the case $\widetilde{E}=\mathbb{R}$ (which trivially happens when $H=0$ ), $\widetilde{E}$ is a discrete (hence at most countable) subset of $\mathbb{R}$ which always includes $t=0-$ in particular $\widetilde{E}=\{0\}$ in the case of the free Schrödinger equation $(V=0)$.

We now apply a version of Trotter formula from the theory of operator semigroups. It is known that $H_{0}=a^{\mathrm{w}}$ is a self-adjoint operator on the maximal domain (see [33])

$$
D\left(H_{0}\right)=\left\{\psi \in L^{2}\left(\mathbb{R}^{d}\right): H_{0} \psi \in L^{2}\left(\mathbb{R}^{d}\right)\right\} .
$$

For our purposes it is enough to assume that $V \in \mathcal{B}\left(L^{2}\left(\mathbb{R}^{d}\right)\right)$, hence we consider bounded perturbations of $H_{0}$. In particular, $V \in L^{\infty}\left(\mathbb{R}^{d}\right)$ is a suitable choice, even for possibly complex-valued potentials.

Then, we have (cf. for instance [12, Cor. 2.7 and Ex. 2.9])

$$
e^{-i t\left(H_{0}+V\right)}=\lim _{n \rightarrow \infty} E_{n}(t), \quad E_{n}(t)=\left(e^{-i \frac{t}{n} H_{0}} e^{-i \frac{t}{n} V}\right)^{n},
$$

with convergence in the strong operator topology in $L^{2}\left(\mathbb{R}^{d}\right)$. We denote by $e_{n, t}(x, y)$ the distribution kernel of $E_{n}(t)$ and by $u_{t}(x, y)$ that of $U(t)=e^{-i t\left(H_{0}+V\right)}$. 
We assume $V \in L^{\infty}\left(\mathbb{R}^{d}\right)$, and we tune its regularity as follows. In view of the discussion on modulation spaces in the previous section, we have available a scale of decreasing regularity spaces.

1. The best option for our purposes is given by $C_{b}^{\infty}\left(\mathbb{R}^{d}\right)$, the space of smooth bounded functions with bounded derivatives of any order.

2. Subsequently we have the (scale of) modulation spaces $M_{s}^{\infty}\left(\mathbb{R}^{d}\right), s>d$, which contain bounded continuous functions becoming less regular as $s \searrow d$ - the parameter $s$ can be thought of as a measure of (fractional) differentiability.

3. We finally consider the Sjöstrand class $M^{\infty, 1}\left(\mathbb{R}^{d}\right)$, where even the partial regularity of the previous level is lost. We are still dealing with bounded continuous functions, which locally enjoy the mild regularity of the Fourier transform of a $L^{1}$ function.

Let us first state our main result at the intermediate regularity encoded by $M_{s}^{\infty}\left(\mathbb{R}^{d}\right)$.

Theorem 2 ([44, Thm. 1.1]) Let $H_{0}=a^{\mathrm{w}}$ as above and $V \in M_{s}^{\infty}\left(\mathbb{R}^{d}\right)$, with $s>2 d$. Let $\mathcal{A}_{t}$ be the classical flow associated with $H_{0}$ as in $(10)$. For any $t \in \mathbb{R} \backslash \widetilde{E}$ :

1. the distributions $e^{-2 \pi i \Phi_{t}} e_{n, t}, n \geq 1$, and $e^{-2 \pi i \Phi_{t}} u_{t}$ belong to a bounded subset of $M_{s}^{\infty}\left(\mathbb{R}^{2 d}\right)$

2. $e_{n, t} \rightarrow u_{t}$ in $\left(\mathcal{F} L_{r}^{1}\right)_{\mathrm{loc}}\left(\mathbb{R}^{2 d}\right)$ for any $0<r<s-2 d$, hence uniformly on compact subsets.

The previous convergence result is expected to improve in the smooth context in view of the characterization given in (8).

Corollary 1 ([44, Cor. 1.2]) Let $H_{0}=a^{\mathrm{w}}$ as above and $V \in C_{b}^{\infty}\left(\mathbb{R}^{d}\right)$. Let $\mathcal{A}_{t}$ denote the classical flow associated with $H_{0}$ as in (10). For any $t \in \mathbb{R} \backslash \widetilde{E}$ :

1. the distributions $e^{-2 \pi i \Phi_{t}} e_{n, t}, n \geq 1$, and $e^{-2 \pi i \Phi_{t}} u_{t}$ belong to a bounded subset of $C_{b}^{\infty}\left(\mathbb{R}^{2 d}\right)$;

2. $e_{n, t} \rightarrow u_{t}$ in $C^{\infty}\left(\mathbb{R}^{2 d}\right)$, hence uniformly on compact subsets together with any derivatives.

It is interesting to compare this result with those obtained by Fujiwara in [26], where convergence at the level of kernels in $C_{b}^{\infty}$-sense for short times was proved. In spite of different assumptions and approximation schemes, we stress that our result is global in time.

We conclude with a convergence result in the same spirit, for potentials in the Sjöstrand class.

Theorem 3 ([44, Thm. 1.3]) Let $H_{0}=a^{\mathrm{w}}$ as discussed above and $V \in M^{\infty, 1}\left(\mathbb{R}^{d}\right)$. Let $\mathcal{A}_{t}$ denote the classical flow associated with $H_{0}$ as in $(10)$. For any $t \in \mathbb{R} \backslash \widetilde{E}$ :

1. the distributions $e^{-2 \pi i \Phi_{t}} e_{n, t}, n \geq 1$, and $e^{-2 \pi i \Phi_{t}} u_{t}$ belong to a bounded subset of $M^{\infty, 1}\left(\mathbb{R}^{2 d}\right)$;

2. $e_{n, t} \rightarrow u_{t}$ in $\left(\mathcal{F} L^{1}\right)_{\mathrm{loc}}\left(\mathbb{R}^{2 d}\right)$, hence uniformly on compact subsets. 
We stress that a typical potential setting in the papers by Albeverio and coauthors is "harmonic oscillator plus a bounded perturbation", the latter in the form of the Fourier transform of a (finite) complex measure on $\mathbb{R}^{d}$ - cf. [1] and the references therein. While those results rely on completely different techniques (in particular, infinitedimensional oscillatory integral operators), in view of the embedding $\mathcal{F} \mathcal{M}\left(\mathbb{R}^{d}\right) \subset$ $M^{\infty, 1}\left(\mathbb{R}^{d}\right)$ proved in [43, Prop. 3.4] we are able to cover this class of potentials too.

In addition to the regularity properties mentioned insofar, our choice of modulation space is particularly well suited to the problem in view of the rich algebraic structure discussed in Section 2. The key of the proofs is that for $t \in \mathbb{R} \backslash \widetilde{E}$ the approximate operator $E_{n}(t)$ can be expressed in integral form and a manageable form of the kernel $e_{n, t}$ can be derived. In particular, with the help of some technical lemmas we are able to write

$$
\begin{aligned}
E_{n}(t) \varphi(x) & =a_{n, t}^{\mathrm{w}} \mu\left(\mathcal{A}_{t}\right) \varphi(x) \\
& =c(t)\left|\operatorname{det} B_{t}\right|^{-1 / 2} \int_{\mathbb{R}^{d}} e^{2 \pi i \Phi_{t}(x, y)} \widetilde{a_{n, t}}(x, y) \varphi(y) d y,
\end{aligned}
$$

where $\Phi_{t}$ is as in $(12)$ and $\left\{a_{n, t}\right\},\left\{\widetilde{a_{n, t}}\right\} \subset M_{s}^{\infty}\left(\mathbb{R}^{2 d}\right)$ are bounded sequences of symbols for fixed $t \in \mathbb{R} \backslash \widetilde{E}$.

\section{Results on integral kernels at exceptional times}

The occurrence of a set of exceptional times in Theorems 2 and 3 comes not as a surprise from a mathematical point of view: it may happen indeed that the integral kernel of the evolution operator degenerates into a distribution. A standard example of this phenomenon is provided by the harmonic oscillator, namely

$$
i \partial_{t} \psi=-\frac{1}{4 \pi} \Delta \psi+\pi|x|^{2} \psi
$$

The integral kernel of the corresponding evolution operator is known as the Mehler kernel and can be explicitly characterized [11, 36]: for $k \in \mathbb{Z}$,

$$
u_{t}(x, y)= \begin{cases}c(k)|\sin t|^{-d / 2} \exp \left(\pi i \frac{x^{2}+y^{2}}{\tan t}-2 \pi i \frac{x \cdot y}{\sin t}\right) & (\pi k<t<\pi(k+1)), \\ c^{\prime}(k) \delta\left((-1)^{k} x-y\right) & (t=k \pi)\end{cases}
$$

for suitable phase factors $c(k), c^{\prime}(k) \in \mathbb{C}$. This shows the expected degenerate behaviour at integer multiples of $\pi$, which is consistent with the fact that the associated classical flow $\mathcal{A}_{t}$ is given by

$$
\mathcal{A}_{t}=\left(\begin{array}{cc}
(\cos t) I & (\sin t) I \\
-(\sin t) I & (\cos t) I
\end{array}\right)
$$


where $I \in \mathbb{R}^{d \times d}$ is the identity matrix. Hence we retrieve $\widetilde{E}=\{t \in \mathbb{R}: \sin t=0\}=$ $\{k \pi: k \in \mathbb{Z}\}$.

We may wonder whether convergence of integral kernels still occurs in some distributional sense, hopefully better than the broadest one (that is $\mathcal{S}^{\prime}\left(\mathbb{R}^{2 d}\right)$ ). In view of the discussion in Section 2 on the triple $\left(M^{1}, L^{2}, M^{\infty}\right)$, a suitable setting may be provided by $M^{\infty}$. We have indeed a general result for the kernels of strongly convergent sequences of operators in $L^{2}$.

Theorem 4 Let $\left\{A_{n}\right\} \subset \mathcal{B}\left(L^{2}\left(\mathbb{R}^{d}\right)\right), n \in \mathbb{N}$, be a sequence of bounded linear operators on $L^{2}\left(\mathbb{R}^{d}\right)$ with associated distribution kernels $\left\{a_{n}\right\} \subset \mathcal{S}^{\prime}\left(\mathbb{R}^{2 d}\right)$, and $A \in \mathcal{B}\left(L^{2}\left(\mathbb{R}^{d}\right)\right)$ with distribution kernel $a \in \mathcal{S}^{\prime}\left(\mathbb{R}^{2 d}\right)$. Assume that $A_{n} \rightarrow A$ in the strong operator topology. Then:

1. $a_{n}, a \in M^{\infty}\left(\mathbb{R}^{2 d}\right), n \in \mathbb{N}$;

2. $a_{n} \rightarrow$ a in the weak-* topology on $M^{\infty}\left(\mathbb{R}^{2 d}\right)$.

In particular we have $a_{n} \rightarrow a$ in $\mathcal{F} L_{\mathrm{loc}}^{\infty}\left(\mathbb{R}^{2 d}\right)$, the latter space endowed with the topology $\sigma\left(\left(\mathcal{F} L^{\infty}\right)_{\text {loc }}\left(\mathbb{R}^{2 d}\right),\left(\mathcal{F} L^{1}\right)_{\text {comp }}\left(\mathbb{R}^{2 d}\right)\right)$.

Proof We have that $\left\{A_{n}\right\}$ is a bounded sequence in $\mathcal{B}\left(L^{2}\left(\mathbb{R}^{d}\right)\right)$ as a consequence of the uniform boundedness principle, hence also in $\mathcal{B}\left(M^{1}\left(\mathbb{R}^{d}\right), M^{\infty}\left(\mathbb{R}^{d}\right)\right)$. The Feichtinger kernel theorem (Theorem 11 yields that the kernels $a_{n}$ belong to a bounded subset of $M^{\infty}\left(\mathbb{R}^{2 d}\right)$. Similarly, $A \in \mathcal{B}\left(L^{2}\left(\mathbb{R}^{d}\right)\right) \Rightarrow a \in M^{\infty}\left(\mathbb{R}^{2 d}\right)$. For the second part of the claim we remark that $A_{n} \rightarrow A$ in the strong operator topology implies that $a_{n} \rightarrow a$ in $\mathcal{S}^{\prime}\left(\mathbb{R}^{2 d}\right)$. Therefore, for any fixed non-zero $g \in \mathcal{S}\left(\mathbb{R}^{d}\right)$ we have $V_{g} a_{n} \rightarrow V_{g} a$ pointwise in $\mathbb{R}^{2 d}$. Moreover, we have the estimate $\left|V_{g} a_{n}(x, \xi)\right| \leq C$, for some constant $C>0$ independent of $n$ by the first part of the claim. Hence, for any $\varphi \in M^{1}\left(\mathbb{R}^{d}\right)$ we have

$$
\begin{aligned}
\left\langle a_{n}, \varphi\right\rangle & =\int_{\mathbb{R}^{2 d}} V_{g} a_{n}(x, \xi) \overline{V_{g} \varphi(x, \xi)} d x d \xi \\
& \rightarrow \int_{\mathbb{R}^{2 d}} V_{g} a(x, \xi) \overline{V_{g} \varphi(x, \xi)} d x d \xi=\langle a, \varphi\rangle,
\end{aligned}
$$

by the dominated convergence theorem.

It would be interesting to prove the boundedness of $a_{n}$ in $M^{\infty}\left(\mathbb{R}^{2 d}\right)$ in Theorem 4 without using the uniform boundedness principle, although it could be not immediate.

A straightforward application of this result allows us to prove global-in-time convergence of integral kernels, although in a weaker sense than before.

Corollary 2 Assume $V \in L^{\infty}\left(\mathbb{R}^{d}\right)$. Let $e_{n, t} \in \mathcal{S}^{\prime}\left(\mathbb{R}^{2 d}\right)$ be the distribution kernel of the Feynman-Trotter parametrix $E_{n}(t)$ in (14) and $u_{t} \in \mathcal{S}^{\prime}\left(\mathbb{R}^{2 d}\right)$ be the kernel of the Schrödinger evolution operator $U(t)$ associated with the Cauchy problem (1). For any $n \in \mathbb{N}$ and $t \in \mathbb{R}$ we have $e_{n, t}, u \in M^{\infty}\left(\mathbb{R}^{2 d}\right)$. Moreover, $e_{n, t} \rightarrow u_{t}$ in the weak-* topology on $M^{\infty}\left(\mathbb{R}^{2 d}\right)$ for any fixed $t \in \mathbb{R}$. 
For more regular potentials we expect that the conclusion of Corollary 2 can be improved. Let us first provide a version of the Trotter formula for potentials in $M^{\infty, 1}\left(\mathbb{R}^{d}\right)$, with strong convergence on $M^{1}\left(\mathbb{R}^{d}\right)$.

Theorem 5 Assume $V \in M^{\infty, 1}\left(\mathbb{R}^{d}\right)$. Let $\left\{E_{n}(t)\right\}$ be the sequence of Feynman-Trotter parametrices defined in (14) and $U(t)$ be the Schrödinger evolution operator $U(t)$ associated with the Cauchy problem (1). For any fixed $t \in \mathbb{R}$ we have

$$
\lim _{n \rightarrow \infty} E_{n}(t)=U(t), \quad \lim _{n \rightarrow \infty} E_{n}(t)^{*}=U(t)^{*}
$$

in the strong topology of operators acting on $M^{1}\left(\mathbb{R}^{d}\right)$.

Proof We prove that $E_{n}(t) \rightarrow U(t)$ strongly in $\mathcal{B}\left(M^{1}\left(\mathbb{R}^{d}\right)\right)$; the claim concerning adjoint operators follows by similar arguments since $U(t)^{*}=U(-t)$ and $E_{n}(t)^{*}=$ $\left(e^{i \frac{t}{n} V} e^{i \frac{t}{n} H_{0}}\right)^{n}$

As already observed, we know that the operator $-i H_{0}$ with domain $D\left(H_{0}\right)=$ $\left\{\varphi \in L^{2}\left(\mathbb{R}^{d}\right): H_{0} \varphi \in L^{2}\left(\mathbb{R}^{d}\right)\right\}$ is self-adjoint $([\overline{33}])$. Let $U_{0}(t)=e^{-i t H_{0}}$ be the corresponding strongly continuous unitary group on $L^{2}\left(\mathbb{R}^{d}\right)$. The well-posedness of the Schrödinger equation $i \partial_{t} \psi=H_{0} \psi$ in $M^{1}\left(\mathbb{R}^{d}\right)$ (see e.g. [9]) implies that the restriction of $U_{0}(t)$ to $M^{1}\left(\mathbb{R}^{d}\right)$ defines a strongly continuous group on $M^{1}\left(\mathbb{R}^{d}\right)$, its generator being the restriction of $H_{0}$ to the subspace $\left\{\varphi \in M^{1}\left(\mathbb{R}^{d}\right): H_{0} \varphi \in\right.$ $\left.M^{1}\left(\mathbb{R}^{d}\right)\right\}$, as a consequence of known results on subspace semigroups, cf. [12, Chapter 2, Sec. 2.3]. Since the pointwise multiplication by $V \in M^{\infty, 1}\left(\mathbb{R}^{d}\right)$ defines a bounded operator on $M^{1}\left(\mathbb{R}^{d}\right)$, the desired result follows from the classical Trotter formula ([12, Cor. 2.7 and Ex. 2.9]).

We provide an equivalent formulation of the previous result for the corresponding integral kernels, which is indeed a partial counterpart of the pointwise convergence results of Section 3 .

Theorem 6 Under the same assumptions of Theorem 3 for all $t \in \mathbb{R}$ and $\varphi \in$ $M^{1}\left(\mathbb{R}^{d}\right)$, the functions

$$
\left\langle e_{n, t}(x, \cdot), \varphi\right\rangle, \quad\left\langle e_{n, t}(\cdot, y), \varphi\right\rangle, \quad\left\langle u_{t}(x, \cdot), \varphi\right\rangle, \quad\left\langle u_{t}(\cdot, y), \varphi\right\rangle
$$

belong to $M^{1}\left(\mathbb{R}^{d}\right)$.

Moreover

$$
\left\langle e_{n, t}(x, \cdot), \varphi\right\rangle \rightarrow\left\langle u_{t}(x, \cdot), \varphi\right\rangle, \quad\left\langle e_{n, t}(\cdot, y), \varphi\right\rangle \rightarrow\left\langle u_{t}(\cdot, y), \varphi\right\rangle
$$

in $M^{1}\left(\mathbb{R}^{d}\right)$, hence in $L^{p}\left(\mathbb{R}^{d}\right)$ for every $1 \leq p \leq \infty$.

The last conclusion follows from the continuous embedding $M^{1}\left(\mathbb{R}^{d}\right) \hookrightarrow L^{p}\left(\mathbb{R}^{d}\right)$, for every $1 \leq p \leq \infty$.

Remark 1 We expect other improvements of Theorem 4 to hold in the case where $A_{n}=E_{n}(t), A=U(t)$. In particular, convergence result for the corresponding 
integral kernels could be investigated in the context of mixed modulation spaces and generalized kernel theorems in the spirit of [7]. We will not engage in such formulation here in order to avoid quite technical discussions.

\section{Physics at exceptional times}

In spite of the attempts to shed light on the nature of exceptional times and the partial results in the previous section, a physical interpretation of exceptional times is still not clear at the moment. This non-trivial question also appears in the form of an enigmatic exercise in the textbook [23, Problem 3-1] by Feynman and Hibbs. While dimensional analysis and heuristic arguments may provide some hints, a precise answer still seems to be missing.

We give our contribution to this discussion with a short argument which elucidates the nature of exceptional times in terms of measurable quantities. Recall that $B(u, r)$ denotes the ball with center $u \in \mathbb{R}^{d}$ and radius $r>0$ in $\mathbb{R}^{d}$. Following the custom in physics we adopt below the bra-ket notation, and we identify states with their wave functions in the position representation.

Fix $x_{0}, y_{0} \in \mathbb{R}^{d}$ and $a, b>0$, and consider the normalised wave-packets

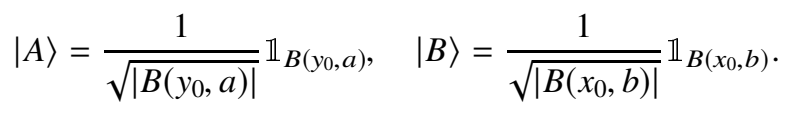

The corresponding transition amplitude from the state $|A\rangle$ to $|B\rangle$ under the Hamiltonian $H=H_{0}+V$ as in Theorem 3 , namely

$$
I=I\left(t, x_{0}, y_{0}, a, b\right)=\langle B|U(t)| A\rangle, \quad t \in \mathbb{R}
$$

trivially satisfies the estimate

$$
\left|I\left(t, x_{0}, y_{0}, a, b\right)\right| \leq 1, \quad \forall t \in \mathbb{R}, x_{0}, y_{0} \in \mathbb{R}^{d}, a, b>0 .
$$

This bound cannot be improved at exceptional times: consider for instance the case where $t=0, x_{0}=y_{0}$ and $a=b$, which yields $I=1$. Nevertheless, we have the following result.

Proposition 3 Under the same assumptions of Theorem 3 for all $t \in \mathbb{R} \backslash \widetilde{E}$ and $x_{0}, y_{0} \in \mathbb{R}^{d}$ we have

$$
\lim _{a, b \rightarrow 0} \frac{I\left(t, x_{0}, y_{0}, a, b\right)}{(a b)^{d / 2}}=C \overline{u_{t}\left(x_{0}, y_{0}\right)}
$$

where $C=C(d)=|B(0,1)|$.

Proof An explicit computation yields 


$$
\frac{I\left(t, x_{0}, y_{0}, a, b\right)}{C(a b)^{d / 2}}=\frac{1}{C^{2}(a b)^{d}} \int_{B\left(x_{0}, b\right)} \int_{B\left(y_{0}, a\right)} \overline{u_{t}(x, y)} d y d x,
$$

and the conclusion follows by the continuity of $u_{t}(x, y)$ in $\mathbb{R}^{2 d}$, because $u_{t} \in$ $\left(\mathcal{F} L^{1}\right)_{\text {loc }}\left(\mathbb{R}^{2 d}\right)$ for $t \in \mathbb{R} \backslash \widetilde{E}$ by Theorem 3 ,

This result shows that while $|I| \leq 1$ in general, for a non-exceptional time $t \in \mathbb{R} \backslash \widetilde{E}$ we have that $|I| \sim(a b)^{d / 2}$ as $a, b \rightarrow 0$. In particular $|I| \rightarrow 0$ as $a, b \rightarrow 0$ except (possibly) for exceptional times.

\section{Acknowledgements}

We would like to express our gratitude to Professors Elena Cordero, Ernesto De Vito and Stephan Waldmann for fruitful conversations on the topics of this paper.

\section{References}

1. S. Albeverio, R. Høegh-Krohn and S. Mazzucchi: Mathematical Theory of Feynman Path Integrals. An Introduction. Lecture Notes in Mathematics 523. Springer-Verlag, Berlin, 2008.

2. S. Albeverio and S. Mazzucchi. Path integral: mathematical aspects. Scholarpedia 6(1):8832, (2011).

3. E. Cordero, H. G. Feichtinger and F. Luef: Banach Gelfand triples for Gabor analysis. In Pseudo-differential Operators, 1-33, Lecture Notes in Math. 1949, Springer, Berlin, 2008.

4. E. Cordero, K. Gröchenig, F. Nicola and L. Rodino: Wiener algebras of Fourier integral operators. J. Math. Pures Appl. 99(2) (2013), 219-233.

5. E. Cordero, K. Gröchenig, F. Nicola and L. Rodino: Generalized metaplectic operators and the Schrödinger equation with a potential in the Sjöstrand class. J. Math. Phys. 55(8) (2014), 081506.

6. E. Cordero, F. Nicola and L. Rodino: Sparsity of Gabor representation of Schrödinger propagators. Appl. Comput. Harmon. Anal. 26 (2009), no. 3, 357-370.

7. E. Cordero and F. Nicola: Kernel theorems for modulation spaces. J. Fourier Anal. Appl. 25 (2019), no. 1, 131-144.

8. E. Cordero and F. Nicola: On the Schrödinger equation with potential in modulation spaces. $J$. Pseudo-Differ. Oper. Appl. 5(3) (2014), 319-341.

9. E. Cordero, F. Nicola and L. Rodino: Schrödinger equations with rough Hamiltonians. Discrete Contin. Dyn. Syst. 35/10 (2015), 4805-4821.

10. E. Cordero, F. Nicola and S. I. Trapasso: Almost diagonalization of $\tau$-pseudodifferential operators with symbols in Wiener amalgam and modulation spaces. J. Fourier Anal. Appl. 25 (2019), no. 4, 1927-1957.

11. M. de Gosson: Symplectic Methods in Harmonic Analysis and in Mathematical Physics. Pseudo-Differential Operators. Theory and Applications. Birkhäuser/Springer Basel AG, Basel, 2011.

12. K.-J. Engel and R. Nagel: A Short Course on Operator Semigroups. Springer, New York, 2006.

13. H. G. Feichtinger: On a new Segal algebra. Monatsh. Math. 92(4) (1981), 269-289.

14. H. G. Feichtinger: Un espace de Banach de distributions tempérées sur les groupes localement compacts abéliens. C. R. Acad. Sci. Paris S'er. A-B, 290(17) (1980), 791-794. 
15. H. G. Feichtinger: Modulation spaces on locally compact abelian groups. Technical report, University of Vienna, 1983.

16. H. G. Feichtinger: Modulation spaces on locally compact Abelian groups. In Proc. Internat. Conf. on Wavelets and Applications, pages 1-56, Chennai, January 2002, 2003. New Delhi Allied Publishers.

17. H. G. Feichtinger and K. Gröchenig: Gabor wavelets and the Heisenberg group: Gabor expansions and short time Fourier transform from the group theoretical point of view. In Wavelets: a tutorial in theory and applications, volume 2 of Wavelet Anal. Appl., pages 359-397. Academic Press, Boston, 1992.

18. H. G. Feichtinger and K. Gröchenig: Gabor frames and time-frequency analysis of distributions. J. Funct. Anal. 146 (1997), no. 2, 464-495.

19. H. G. Feichtinger and M. S. Jakobsen: Distribution theory by Riemann integrals. Mathematical Modelling, Optimization, Analytic and Numerical Solutions (2020), pages 33-76.

20. H.G. Feichtinger and G. Zimmermann: A Banach space of test functions for Gabor Analysis. In Gabor Analysis and Algorithms, 123-170, Appl. Numer. Harmon. Anal., Birkhäuser Boston, Boston, MA, 1998

21. R. Feynman: Space-time approach to non-relativistic Quantum Mechanics. Rev. Mod. Phys. 20 (1948), 367-387.

22. R. Feynman: Space-time approach to quantum electrodynamics. Phys. Rev. (2) 76 (1949), 769-789.

23. R. Feynman and A.R. Hibbs: Quantum Mechanics and Path Integrals. Emended Edition. Dover Publications, Mineola, 2005.

24. G. B. Folland: Harmonic Analysis in Phase Space. Annals of Mathematics Studies, 122. Princeton University Press, Princeton, NJ, 1989.

25. D. Fujiwara: A construction of the fundamental solution for the Schrödinger equation. J. Anal. Math. 35:41-96, 1979.

26. D. Fujiwara: Remarks on convergence of some Feynman path integrals. Duke Math. J. 47 (1980), 559-600.

27. D. Fujiwara: Rigorous Time Slicing Approach to Feynman Path Integrals. Springer, Tokyo, 2017.

28. K. Gröchenig: Foundations of Time-frequency Analysis. Applied and Numerical Harmonic Analysis. Birkhäuser Boston, Inc., Boston, MA, 2001.

29. K. Gröchenig: Time-frequency analysis of Sjöstrand's class. Rev. Mat. Iberoam. 22(2) (2006), $703-724$.

30. K. Gröchenig and Z. Rzeszotnik: Banach algebras of pseudodifferential operators and their almost diagonalization. Ann. Inst. Fourier 58 (2008), no. 7, 2279-2314.

31. K.Gröchenig and T. Strohmer: Pseudodifferential operators on locally compact abelian groups and Sjöstrand's symbol class. J. Reine Angew. Math. 613 (2007), 121-146.

32. C. Grosche and F. Steiner: Handbook of Feynman path integrals. Springer, Berlin, 1998.

33. L. Hörmander: Symplectic classification of quadratic forms, and general Mehler formulas. Math. Z. 219 (1995), no. 3, 413-449.

34. L. Hörmander: The Analysis of Linear Partial Differential Operators III. Pseudo-differential Operators. Reprint of the 1994 edition. Classics in Mathematics. Springer, Berlin, 2007.

35. M.S. Jakobsen: On a (no longer) new Segal algebra: a review of the Feichtinger algebra. $J$. Fourier Anal. Appl. 24 (2018), no. 6, 1579-1660.

36. L. Kapitanski, I. Rodnianski and K. Yajima: On the fundamental solution of a perturbed harmonic oscillator. Topol. Methods Nonlinear Anal. 9(1) (1997), 77-106.

37. J. R. Klauder: A Modern Approach to Functional Integration. Birkhäuser, Boston MA, 2011.

38. H. Kleinert: Path Integrals in Quantum Mechanics, Statistics and Polymer Physics. World Scientific, Singapore, 1995.

39. S. Mazzucchi: Mathematical Feynman Path Integrals and Their Applications. World Scientific Pub. Co. Inc., 2009.

40. E. Nelson: Feynman integrals and Schrödinger equation. J. Math. Phys. 5 (1964), 332-343.

41. F. Nicola: Convergence in $L^{p}$ for Feynman path integrals. Adv. Math. 294 (2016), 384-409. 
42. F. Nicola: On the time slicing approximation of Feynman path integrals for non-smooth potentials. J. Anal. Math. 137(2) (2019), 529-558.

43. F. Nicola and S. I. Trapasso: Approximation of Feynman path integrals with non-smooth potentials. J. Math. Phys. 60 (2019), 102103.

44. F. Nicola and S. I. Trapasso: On the pointwise convergence of the integral kernels in the Feynman-Trotter formula. Comm. Math. Phys. (2019) - DOI: 10.1007/s00220-019-03524-2, to appear.

45. M. Reed and B. Simon: Methods of Modern Mathematical Physics. Vol. I: Functional analysis. Academic Press, 1981.

46. M. Reed and B. Simon: Methods of Modern Mathematical Physics. Vol. II: Fourier analysis, self-adjointness. Elsevier, 1975.

47. M. Reich and W. Sickel: Multiplication and composition in weighted modulation spaces. In Mathematical analysis, probability and applications - plenary lectures, 103-149, Springer Proc. Math. Stat., 177, Springer, [Cham], 2016.

48. W. Rudin: Functional Analysis. Second edition. International Series in Pure and Applied Mathematics. McGraw-Hill, New York, 1991.

49. J. Sjöstrand: An algebra of pseudodifferential operators. Math. Res. Lett. 1(2) (1994), 185-192.

50. S. I. Trapasso: A time-frequency analysis perspective on Feynman path integrals. arXiv:2004.01784

51. M. W. Wong: Weyl Transforms. Universitext. Springer-Verlag, New York, 1998. 